\title{
La importancia de los MOOC en la educación universitaria
}

The importance of MOOCs in university education

Recibido el 14 de agosto del 2017, aceptado el 16 de octubre del 2017

Asencios Trujillo, Lucia lucia_asencios@hotmail.com Universidad Nacional Enrique Guzmán y Valle - Perú ORCID: 0000-002-4438-1488
Para referenciar este artículo: Asencios, L. (2017). La importancia de los MOOC en la educación universitaria. ConCiencia EPG, 2(2), 75-79.

\section{Resumen}

$\mathrm{E}$ n el artículo se describen la importancia de los MOOC en el sistema educativo de todos los niveles, se hace referencia a la falta de interés en nuestro medio, sobre todo en las instituciones públicas, de querer implementar este sistema, está comprobado que es una alternativa para muchos estudiantes y profesionales, sin embargo, la falta de conocimientos de las autoridades o el desinterés hace que aún no se implementen en muchas instituciones académicas. Se describen las características más resaltantes de los MOOCs las cuales hacen referencia de las bondades de este sistema. También se describe la importancia para el aprendizaje en la sociedad del conocimiento y, finalmente se da a conocer algunas de las instituciones que contribuyen en el aprendizaje a través de su plataforma o uso del MOOC. Con lo cual le hace más conocidos en cualquier parte del mundo, debido a su característica de modalidad, rapidez, y mejor sin ellos.

Palabras clave: $M O O C$, educación, aprendizaje interactivo, aprendiza online, aprendizaje abierto

\section{Summary}

Whe article describes the importance of MOOCs in the educational system at all levels, it refers to the lack of interest in our environment, especially in public institutions, to implement this system, it is proven that it is an alternative for Many students and professionals, however, in our environment there are still gaps in knowledge and educational practices through this system. Therefore, the most outstanding characteristics of the MOOCs are described, which refer to the benefits in the educational field. The importance for learning in the knowledge society is also described and, finally, some of the institutions that contribute to learning through its platform or use of the MOOC are made known, with which, they 
become more known in the world, due to its modality, speed and efficiency.

Keywords: MOOC, education, interactive learning, online learning, open learning

\section{Introducción}

$\mathrm{E}$ n la actualidad el uso de la tecnología se ha convertido en una herramienta imprescindible para nuestras vidas; dado que, gracias a ello podemos adquirir, intercambiar culturas y compartir conocimientos a través de las plataformas virtuales desde cualquier parte del mundo de manera casi inmediata. Son factores característicos de esta era digital a la que estamos sumergidos y que influye de manera directa en el panorama educativo. Existen muchos programas a distancia que son impartidos por diversas instituciones, dado que, no solo ofrece rapidez o inmediatez en la clase, sino que muestran la eficiencia y eficacia en calidad educativa (García, 2017), brindan la oportunidad de adquirir conocimientos según la necesidad que demande el estudiante.

Los cursos abiertos y en línea conocidos como "MOOC" (Curso en línea abierto masivo) es una revolución con un gran potencial en el mundo educativo y formativo (Aguaded, Vázquez \& Sevillano, 2013). Entre sus características que más atrae a la gente o a los estudiantes de todo el planea, destacan: el carácter abierto (OPEN), su capacidad de ubicar la información y la relación entre los distintos actores educativos en internet (ONLINE) y el hecho de que es capaz de abarcar la formación de miles de personas (MASSIVE) al mismo tiempo (Méndez, 2013).

Se pueden discriminar varios tipos de MOOC, algunos de ellos se diferencian en los objetivos, metodologías y resultados. En la actualidad se distinguen entre: xMOOC, se basan en cursos universitarios tradicionales que parecen reproducir la pedagogía de la tecnología del aula. Según Martí (2012), son de los que últimamente se está hablando más a nivel educativo. Normalmente son llevados a cabo por profesores universitarios basados en la adquisición de contenidos y en un modelo de evaluación muy parecido a las clases tradicionales (con unas pruebas más estandarizadas y concretas), presentan una serie de pruebas automatizadas y poseen una gran difusión mediática. Por su parte los cMOOC, se basan en el aprendizaje conectivista, donde el profesor toma el rol de facilitador inicial y los estudiantes lo completan e interactúan con los otros participantes, creando blogs, redes sociales, etc., (Downes, 2011).

Si la influencia de las MOOC es tan importante en el aprendizaje, en el Perú, no se utiliza de forma constante como en otros países, y por lo tanto un gran porcentaje de la población de estudiantes ignoran el valor de la propuesta. Este desconocimiento lleva a muchos a quedarse sin estudios, a lo no capacitación. Por ello, en esta comunicación tratamos de describir la importancia que tiene esta modalidad para la educación contemporánea.

\section{MOC y aprendizaje interactivo}

Los MOOC surgieron como respuesta a los retos actuales de educación las instituciones tienen la necesidad de capacitar a una población que busca educación de calidad a bajo costo (Tamez, 2014) y que, además, ahorre tiempos, viajes, transporte, estadías, y otros recursos.

Según el informe del 2016 de "Curso masivo en línea abierto (MOOC) 2016-2020" hace referencia que: los Massive Open Online Courses, representa un enfoque basado en la distancia a elearning en el que muchos estudiantes pueden participar en una manera altamente colaborativa e interactiva. El contenido del curso se distribuye utilizando la plataforma web bajo un modelo por curso o de suscripción. Hay algunos factores claves del mercado a considerar con MOOC incluyendo la certificación de bajo costo, aprovechando diversas tecnologías (datos en todas partes, teléfonos inteligentes, tabletas y proliferación de dispositivos portátiles, experiencia de aprendizaje flexible, etc.), reducción de costos para capacitación corporativa y otros. 
Se puede argumentar que uno de los principales motores del crecimiento de la industria MOOC actual es la reducción de costos para los programas de aprendizaje y desarrollo en grandes corporaciones. Importante para el ecosistema de las TIC, entre otros.

\section{Características de los MOOC}

Las características puntales de este sistema son: de carácter abierto (OPEN), su capacidad de ubicar la información y la relación entre los distintos actores educativos en internet (ONLINE) y por el hecho de que es capaz de abarcar la formación de miles de personas (MASSIVE) (Méndez, 2013).

Además, Gea (2015) menciona que generalmente este sistema se basa en: ser un curso, es decir cumplir con los requisitos de programación de contenidos, evaluación, cronograma de desarrollo, etc.; estar en línea; tener carácter masivo; tener alcance global, e estar diseñado para un lugar específico; y, abierto, que todos los que desean aprender sobre el tema puedan participar.

\section{Plataformas MOC para docentes}

En la actualidad existe una gran cantidad de instituciones que brindan este servicio orientado específicamente al personal docente. Entre ellas tenemos:

MOOC del INTEF: El (Instituto Nacional de Tecnologías Educativas y de Formación del Profesorado) es una entidad dependiente del Ministerio de Educación de España, y en los últimos años ha implementado su propia plataforma de cursos online y a distancia pensados para docentes:

Miríada X: Nace como una plataforma en la que aunar el conocimiento a través de MOOCs de "universidades e instituciones iberoamericanas", con lo que su principal idioma es el español.

UNED abierta: La Universidad Nacional de Educación a Distancia de España tiene su portal UNED abierta, un compendio con todos sus cursos con los que buscan "abrir el conocimiento" al mundo entero.

Iversity: Tiene cursos online al alcance de todos, su propuesta principal es la participación o de trabajo en grupo, con el objetivo de mejorar las presentaciones en público y tener una buena exposición por parte del docente.

Adámica: Desde Buenos Aires, Argentina, ofrece cursos principalmente técnicos diferenciados en niveles (iniciación, intermedio, avanzado y experto), con etiquetas para separar contenidos y una interfaz muy cómoda y fácil de utilizar.

Udacity: Es una propuesta, general mente sobre conocimientos relacionados con la industria tecnológica en el que resaltan diseños (web, de apps en Android o iOS) o del machine learning, etc., pero también imparten cursos de matemáticas (estadística, álgebra), psicología, marketing, etc.

Harvard Open Courses: La prestigiosa Universidad de Harvard, en Boston, tiene todo tipo de cursos online dentro de lo que llaman Harvard Open Courses. Algunos gratuitos y otros incorporados como cursos oficiales, intercambiables por créditos.

Coursera: es una de las webs más completas que existen, imparten todo tipo de conocimientos, desde los tecnológicos (análisis y estadística en Excel o R, programación en Python), educativos y de formación (aprendiendo a aprender, aprendizaje automático, herramientas de aprendizaje), ciencias de la salud, sociales o casi, entre otros.

Udemy: A diferencia de Coursera, en Udemy también se puede lanzar tu propio curso, con una descriptición clara en cuanto a estrategias, programación temática, cronograma, y sobre todo, el costo. Udemy es de pago, y los cursos de especialización merece la pena desarrollarla.

Khan Academy: En 2006, Salman Khan fundó Khan Academy, una comunidad de conocimiento online constituida como organización sin ánimo 
de lucro y con el objetivo de proporcionar una educación a cualquier persona, en cualquier lugar.

$E d X$ : Las mejores universidades del mundo están registradas en edX, donde ponen a disposición de todo el mundo cientos de cursos sobre materias de nivel universitario, para docentes y con todo tipo de temáticas a tratar.

Stanford MOOC: Una de las mejores universidades del mundo y propone decenas de cursos incluyendo cursos de tipo técnico, médico, etc., y está al alcance de todos gracias a su plataforma de cursos completamente gratuitos.

MIT OpenCourseware: La plataforma de cursos abiertos del Instituto de Tecnología de Massachusetts se llama MIT OpenCourseware, un repositorio con decenas de cursos sobre todas las temáticas habidas y por haber, entre ellas la educación en diferentes ámbitos y con varios objetivos. Aunque muchos de los cursos ya se realizaron, los materiales que se utilizaron están publicados en la respectiva web del curso y puede ser realmente interesante para aprender de ellos.

CMU Open Learning Initiative: La Carnegie Mellon University tiene una plataforma, Open Learning Initiative, con multitud de cursos.
Open Learning: Es una plataforma de cursos y MOOCs a distancia, ofrecen todo tipo de temas y cuestiones, hay cursos dedicado a educación en el que se puede compartir experiencias a través de los cursos.

UPVX: Lo gestiona la Universidad Politécnica de Valencia y han puesto en marcha cursos online masivos y abiertos. Además, podemos encontrar entre otros temas material de interés para la comunidad educativa y para la formación docente.

\section{Conclusiones}

En la última década, los MOOC han producido cambios fuertes en la enseñanza n y aprendizaje de los estudiantes, si bien es cierto nace como una alternativa a la educación tradición, en la actualidad se ha convertido en la primera opción de estudio, debido a que la mayoría trabajan y es muy difícil coincidir horarios que no se crucen con tu actividad académico ante ello, los MOOCs es una opción casi única para seguir profundizando en el conocimiento en el campo académico. En nuestro medio hay una gran deficiencia sobre todo desde las instituciones públicas por implementar este sistema, debido al bajo conocimiento que tienen sobre esta alternativa. 


\section{Referencias}

Aguaded, I., Vázquez, E. \& Sevillano, M. (2013). MOOC, ¿turbocapitalismo de redes o altruismo educativo? Hacia un modelo más sostenible. Informe Scopeo, 2. MOOC: Estado de la situación actual, posibilidades, retos y futuro. Universidad de Salamanca. Recuperado de http://scopeo.usal.es/wpcontent/uploads/2013/06/scopeoi002.pdf

Curso en línea abierto masivo (MOOC) 2016 - 2020; lanzamiento de un nuevo informe (2016, 22 de febrero). M2 Presswire. Recuperado de https://www.search.proquest. $\mathrm{com} /$ docview $/ 1767057388$ ? accountid $=43847$

Downes, S. (2011). Connectivism and connective knowledge. Huffington Post - Education. Recuperado de http://www.huffingtonpost. com/stephen-downes/connectivismandconnecti_b_804653.html.

García, L. (2017). Educación a distancia y virtual: calidad, disrupción, aprendizajes adaptativo y móvil. RIED. Revista Iberoamericana de Educación a Distancia, 20(2), pp. 09-25. doi: http://dx.doi.org/10.5944/ried.20.2.18737

Gea, M. (2015). Informe MOOC y criterios de calidad. Recuperado de http://www. crue.org/TIC/Documents/InformeMOOC_ CRUETIC_ver1 0.pdf

Martí, J. (2012) Tipos de MOOCs. Recuperado de http://www.xarxatic.com/tipos-de-moocs/

Méndez, C. (2013). Diseño e implementación de cursos abiertos masivos en línea (MOOC): expectativas y consideraciones prácticas. RED, Revista de Educación a Distancia, 39. Recuperado de http://www.um.es/ead/ $\mathrm{red} / 39$

Tamez, M. (2014). MOOC. Observatorio de la Innovación Educativa del Tecnológico de Monterrey. Recuperado de http://www.sitios. itesm.mx/webtools/Zs2Ps/roie/mayo14.pdf 